\title{
Preliminary RNA-microarray analysis of long non-coding RNA expression in abnormally invasive placenta
}

\author{
HUISHAN ZHANG ${ }^{1}$, SHUZHEN WU ${ }^{2}$, SHAOXIN YE ${ }^{1,2}$, HUITING MA $^{1}$ and ZHENGPING LIU ${ }^{1,2}$ \\ ${ }^{1}$ Department of Fetal Medicine Research, Foshan Fetal Medicine Research Institute; ${ }^{2}$ Department of Obstetrics, Foshan \\ Women and Children's Hospital Affiliated to Southern Medical University, Foshan, Guangdong 528000, P.R. China
}

Received April 14, 2020; Accepted August 26, 2020

DOI: $10.3892 /$ etm.2020.9445

\begin{abstract}
Long non-coding RNAs (lncRNAs) are reported to have important roles in placental development and function, but the role of lncRNAs in abnormally invasive placenta (AIP) remains elusive. In the present study, the differential expression profiles of IncRNAs were analyzed to identify novel targets for further study of AIP. A total of 10 lncRNAs were chosen for validation by reverse transcription-quantitative PCR. To further determine the functions of dysregulated lncRNAs and their corresponding mRNAs, functional enrichment analysis, coexpression analysis were performed. A total of 329 lncRNAs and 179 mRNAs were identified to be differently expressed between the invasive and control group. Gene ontology analysis revealed that the 10 most significantly enriched functions included upregulated mRNAs and the most significantly enriched term was related to the proteinaceous extracellular matrix (ECM). In the pathway analysis, the two most significantly enriched pathways were the TGF- $\beta$ signaling pathway for upregulated mRNAs and the pentose phosphate pathway for downregulated mRNAs. Furthermore, for certain dysregulated lncRNAs, their associated mRNAs were also dysregulated. Of note, BMP and activin membrane-bound inhibitor and TGF- $\beta$-induced, as the target genes of the TGF- $\beta$ pathway, were indicated to be closely related to the ECM and invasive placental cells. Their nearby lncRNAs G008916 and vault RNA2-1 were also significantly dysregulated. In conclusion, significant lncRNAs with the potential to serve as biomarkers for AIP were identified.
\end{abstract}

Correspondence to: Dr Zhengping Liu, Department of Obstetrics, Foshan Women and Children's Hospital Affiliated to Southern Medical University, 11 Renminxi Road, Foshan, Guangdong 528000, P.R. China

E-mail: liuzphlk81@outlook.com

Key words: long non-coding RNAs, mRNAs, expression profile, abnormally invasive placenta

\section{Introduction}

Abnormally invasive placenta (AIP) is a pathologic condition in which the placenta abnormally attaches to and invades the uterine wall (1). AIP is regarded as one of the most dangerous conditions associated with pregnancy. The main complication of AIP is massive hemorrhaging when attempts are made to remove the placenta manually during delivery. Massive hemorrhaging may result in multisystem organ failure, disseminated intravascular coagulation, hysterectomy, intensive care requirement and even death (2). AIP is a common complication in obstetrics and it is the leading cause of hysterectomies associated with caesarean delivery and peripartum hysterectomies (3). A standardized approach with a comprehensive multidisciplinary care team accustomed to the management of AIP is essential for favourable maternal and neonatal outcomes (4-6). The early diagnosis of pregnant females with AIP will provide more opportunities to receive multidisciplinary care and good perinatal outcomes (7). Studies have indicated that certain placental and fetal hormones, such as human chorionic gonadotropin, alpha-fetoprotein and pregnancy-associated plasma protein A, are helpful in the diagnosis of AIP $(8,9)$. However, none of the placental/fetal proteins have proved useful for diagnosing AIP earlier than ultrasound and MRI. The major prenatal diagnostic methods of AIP are still ultrasound and MRI examination. AIP remains unpreventable and at present, more than half of all pregnant females with AIP remain undiagnosed after imaging examinations prior to delivery (10).

Although AIP may be diagnosed through imaging examinations as well as placental and fetal hormones, the expression of lncRNAs (long non-coding RNAs) and the roles they have in AIP have remained elusive. lncRNAs are transcripts no longer than $200 \mathrm{bp}$, which were indicated to actively regulate the expression and function of thousands of protein-coding genes through various mechanisms (11). Numerous lncRNAs identified in the human placenta are reported to be involved in preeclampsia and intrauterine growth restriction due to their regulation of trophoblast differentiation, proliferation and migration (12). IncRNA metastasis-associated adenocarcinoma transcript 1 was even proved to be involved in AIP and associated with trophoblast-like cell invasion (13). These studies imply that lncRNAs may participate in the process of AIP and have great potential to serve as biomarkers of AIP. 
Reliable biomarkers for prediction and diagnosis may contribute to perinatal outcomes. Thus, it was attempted to seek potential and valid lncRNAs to serve as biomarkers for the efficient and precise prediction and diagnosis of AIP. In the present study, the lncRNA expression profiles of patients with AIP were determined and 5 randomly selected lncRNAs were validated by reverse transcription-quantitative (RT-q)PCR.

\section{Materials and methods}

Sample collection and ethics approval. In the present study, placenta tissues from pregnant females enrolled at Southern Medical University Affiliated Maternal \& Child Health Hospital of Foshan (Foshan, China) between December 2017 and June 2018 were analysed. A total of 5 pregnant females who were antenatally suspected of having AIP according to array-scale and doppler sonography were enrolled in the study. All of these 5 patients were diagnosed with AIP by expert surgeons during the operation according to the clinical grading system (14). Subsequently, the diagnosis was confirmed through pathological examination according to the pathological classification criteria (15). Clinical and demographic data on each of the patients is provided in Table I. All pregnant females were diagnosed with a scarred uterus and placenta previa, and they had no hypertension, diabetes or other obstetric complications. Tissues from each patient of the AIP group included one sample from the invasive site and another sample from the adherent noninvasive site of the placenta according to the operators' instructions. They were divided into the invasive and control group. In addition, for extended sample validation, placental tissues were collected from 15 patients with AIP and 15 patients with a normal placenta. The two groups were referred to as the AIP group and the normal group. After placental delivery, all of the samples were cut from the surface of the maternal placenta. They were washed in PBS five times to remove blood cells and were then transferred into liquid nitrogen within $10 \mathrm{~min}$.

The Human Ethics Committee of the Foshan Women and Children's Hospital Affiliated to Southern Medical University (Foshan, China) approved this study (approval no. FSFY-MEC-2017-055). Written informed consent was obtained from all pregnant females for using their specimens and other clinical information. All of the methods were performed according to the approved guidelines of the Human Ethics Committee.

RNA extraction, amplification and hybridization. For microarray analysis, lncRNAs and mRNAs were detected with the Arraystar Human LncRNA Microarray v4.0 (Arraystar, Inc.). Following the manufacturer's protocol, total RNA was extracted from frozen samples using TRIzol (Invitrogen; Thermo Fisher Scientific, Inc.). The RNA quantity and quality were measured using a NanoDrop ND-1000 (Thermo Fisher Scientific, Inc.). An Agilent 2100 Bioanalyzer (Agilent Technologies, Inc.) and standard denaturing agarose gel electrophoresis were used to evaluate RNA integrity. Following the Agilent One-Color Microarray-Based Gene Expression Analysis protocol (Agilent Technologies, Inc.), array hybridization and sample labelling were performed. After removing ribosomal RNA and performing amplification, $100 \mathrm{ng}$ of total
RNA was labelled and hybridized. Subsequently, the hybridized arrays were washed, fixed and scanned on the Agilent DNA Microarray Scanner. The expression data were stored in the Gene Expression Omnibus database (https://www.ncbi. nlm.nih.gov/geo) under the accession number GSE126552.

$R T-q P C R$ validation. In the present study, original validation (samples were divided into invasive group and control group, and all sample were collected from 5 patients with AIP) and extended validation (samples were divided into AIP group and normal group. Samples of AIP group were collected from 15 patients with AIP, samples of the normal group were collected from 15 patients without AIP) was conducted to validate the results of microarray through RT-qPCR. For RT-qPCR validation, total RNA was extracted with TRIzol reagent from the remaining portion of tissues not used in the lncRNA microarray. Subsequently, first-strand complementary DNAs were generated using SuperScript ${ }^{\mathrm{TM}}$ III Reverse Transcriptase (Invitrogen; Thermo Fisher Scientific, Inc.) following the manufacturer's instructions. The RT-qPCR process was performed using a ViiA-7 RT-PCR System (Applied Biosystems; Thermo Fisher Scientific, Inc.) and 2X PCR master mix (Arraystar, Inc.). The relative expression levels of lncRNAs were quantified using the $2^{-\Delta \Delta \mathrm{Cq}}$ method (16) and were normalized to $\beta$-actin expression. The primers for RT-qPCR were designed according to the lncRNA sequences in NONCODE (http://www.noncode.org) and primer sequences were synthesized and purified by Yingjun Co. The primer sequences are listed in Table SI.

Bioinformatics analysis of RNA scanned data. Raw data were extracted from the acquired array images using Agilent Feature Extraction software 11.0.1.1. Subsequently, GeneSpring GX v12.1 software (Agilent Technologies, Inc.) was used to normalize quantiles of raw data. IncRNAs and mRNAs with flags in 'Present' or 'Marginal' ('All Targets Value') in at least 5 out of 10 samples were chosen for further data analysis. Dysregulated lncRNAs and mRNAs between two groups with a fold change $>1.5$ were filtered based on their P-value/false discovery rate $(<0.05)$.

Gene Ontology (GO) and Kyoto Encyclopaedia of Genes and Genomes (KEGG) pathway analyses were applied to predict the roles of the dysregulated mRNAs by determining GO terms and biological pathways, respectively. In-house scripts of R3.5.0 were used to perform hierarchical clustering and to perform combined analysis. First, http://bioconductor.org/biocLite.R was sourced to install the clusterProfiler package and load org.Hs.eg.db. Subsequently, the function of enrichGO and enrichKEGG was used to obtain the results.

Coexpression analysis of the dysregulated lncRNAs subgroup was also performed to identify coregulated lncRNA-mRNA pairs. The subgroups of dysregulated RNA coregulation pairs that were dysregulated included large intervening noncoding RNAs (lincRNAs), antisense lncRNAs and their paired dysregulated mRNAs.

Statistical analysis. In present study, all subsequent analysis was conducted using R 3.5.0 (https://www.r-project.org/). Proportions or mean \pm standard error were used to present 
Table I. Clinical details of pregnant females.

\begin{tabular}{|c|c|c|c|c|c|c|}
\hline No. & Age (years) & $\begin{array}{l}\text { Gestational age } \\
\text { (weeks) }\end{array}$ & $\begin{array}{c}\text { Prior caesarean } \\
\text { section }\end{array}$ & $\begin{array}{l}\text { Other uterine } \\
\text { surgery }\end{array}$ & Hysterectomy & $\begin{array}{l}\text { Histological } \\
\text { grade }^{\mathrm{a}}\end{array}$ \\
\hline 1 & 29 & $36+1$ & 1 & 2 (induced abortion) & No & Percreta \\
\hline 2 & 30 & $34+5$ & 1 & 0 & No & Increta \\
\hline 3 & 28 & $35+1$ & 1 & 1 (induced abortion) & No & Increta \\
\hline 4 & 30 & $36+1$ & 1 & 0 & No & Increta \\
\hline 5 & 29 & $35+4$ & 1 & 0 & No & Accreta \\
\hline
\end{tabular}

aPlacenta accreta was defined as the villi simply adhering to the myometrium. Placenta increta was diagnosed when the villi invaded the myometrium, while placenta percreta was defined as the villi invading the full thickness of the myometrium, including the uterine serosa and at times adjacent pelvic organs.

all variables when appropriate. A Mann-Whitney U test was used to compare differences in RT-qPCR results between invasive group and control group and a Mann-Whitney U test was used to compare differences in RT-qPCR results between the AIP group and normal group. $\mathrm{P}<0.05$ was considered to indicate a statistically significant difference.

\section{Results}

Expression profile of lncRNAs and mRNAs. To explore the lncRNA and mRNA expression profiles in AIP, 5 pairs of placenta tissues of patients with AIP were screened and divided into two groups: The invasive group and the control group. In total, 20,730 mRNAs and 40,173 lncRNAs were detected by microarray analysis. Among them, 329 lncRNAs were significantly differentially expressed (fold change $>1.5$, $\mathrm{P}<0.05)$. Of the dysregulated lncRNAs, 101 transcripts were upregulated, while 228 transcripts were downregulated in the invasive group. The top dysregulated lncRNAs in the invasive group were leucine-rich repeat-containing 38 among the upregulated lncRNAs and ELMO domain-containing 1 among the downregulated lncRNAs. Furthermore, a total of 179 dysregulated mRNAs (fold change $>1.5, \mathrm{P}<0.05$ ) were identified. Of the dysregulated mRNAs in the invasive group, 95 transcripts were upregulated and 84 transcripts were downregulated. The most significantly dysregulated mRNAs in the invasive group were AF003625.3 (upregulated) and G037111 (downregulated). Hierarchical clustering was performed for inter-group comparisons of differential gene expression patterns (Fig. 1A and B).

Expression signatures of the dysregulated lncRNAs and coexpression analysis of subgroup. To further understand lncRNA expression patterns in AIPs, the length, chromosome and classification distribution of the dysregulated lncRNAs were also analysed (Fig. 2). lncRNAs were divided into six categories according to their association with protein-coding genes. The categories were bidirectional, intergenic, exon sense-overlapping, intron sense-overlapping, natural antisense and intronic antisense. The statistical results indicated that the majority of dysregulated lncRNAs were intergenic (72.15\%; Fig. 2B) and were 400-800 nt in length $(69.30 \%$; Fig. 2C). The chromosome distribution indicated that chromosome $2(9.42 \%)$ and $10(6.69 \%)$ contained most of the differentially expressed lncRNAs (Fig. 2A). The expression profile of the lncRNAs revealed dysregulated lncRNAs that may take part in AIP.

A coexpression analysis was used to further determine the potential functions of differentially expressed lncRNA subgroup in AIP. A total of 10 pairs of lincRNA-mRNA and 2 pairs of antisense lncRNA-mRNA (distance $<300 \mathrm{~kb}$ ) were indicated to be coregulated transcripts (Table II).

$R T-q P C R$ results. To independently validate the dysregulated lncRNAs detected by the RNA-microarray, 10 aberrantly expressed lncRNAs [AC009499.1, FOXP4 antisense RNA 1 (FOXP4-AS1), RP5-875H18.4, long intergenic non-protein coding RNA 221 (LINC00221), ATP binding cassette subfamily C member 6 pseudogene 2 (ABCC6P2), G037111, CTD-2311B13.2, G033813, G008916 and vault RNA 2-1 (VTRNA2-1)] were randomly selected. Their expression was validated by RT-qPCR. The validation results demonstrated that AC009499.1, FOXP4-AS1, RP5-875H18.4, G037111, CTD-2311B13.2, G033813 and VTRNA2-1 were downregulated, while LINC00221, ABCC6P2 and G008916 were upregulated in the invasive group and AIP group vs. the control and normal group $(\mathrm{P}<0.05$; Fig. $3 \mathrm{~A}$ and $\mathrm{B})$. In addition, the fold changes of the 10 lncRNAs in both original and extended validation were close to the results of the microarray (Fig. 3C). In summary, the RT-qPCR results exhibited good consistency with the RNA-microarray data, indicating that to determine lncRNA changes, RNA-microarray methodology has good reliability and reproducibility.

Functional enrichment analysis. GO and KEGG pathway analyses were performed to identify the functional roles of differentially expressed mRNAs in AIPs. GO analysis was performed in three domains: Biological process (BP), cellular component (CC) and molecular function (MF). The results suggested that all 10 of the most significant functions were enriched with upregulated mRNAs. As presented in Fig. 4A, in the BP domain, the upregulated genes were most enriched in the terms proteinaceous extracellular matrix (GO:0005578), extracellular matrix (ECM; GO:0031012) and ECM component (GO:0044420). For CC and MF, the upregulated genes were most enriched in multicellular organismal 

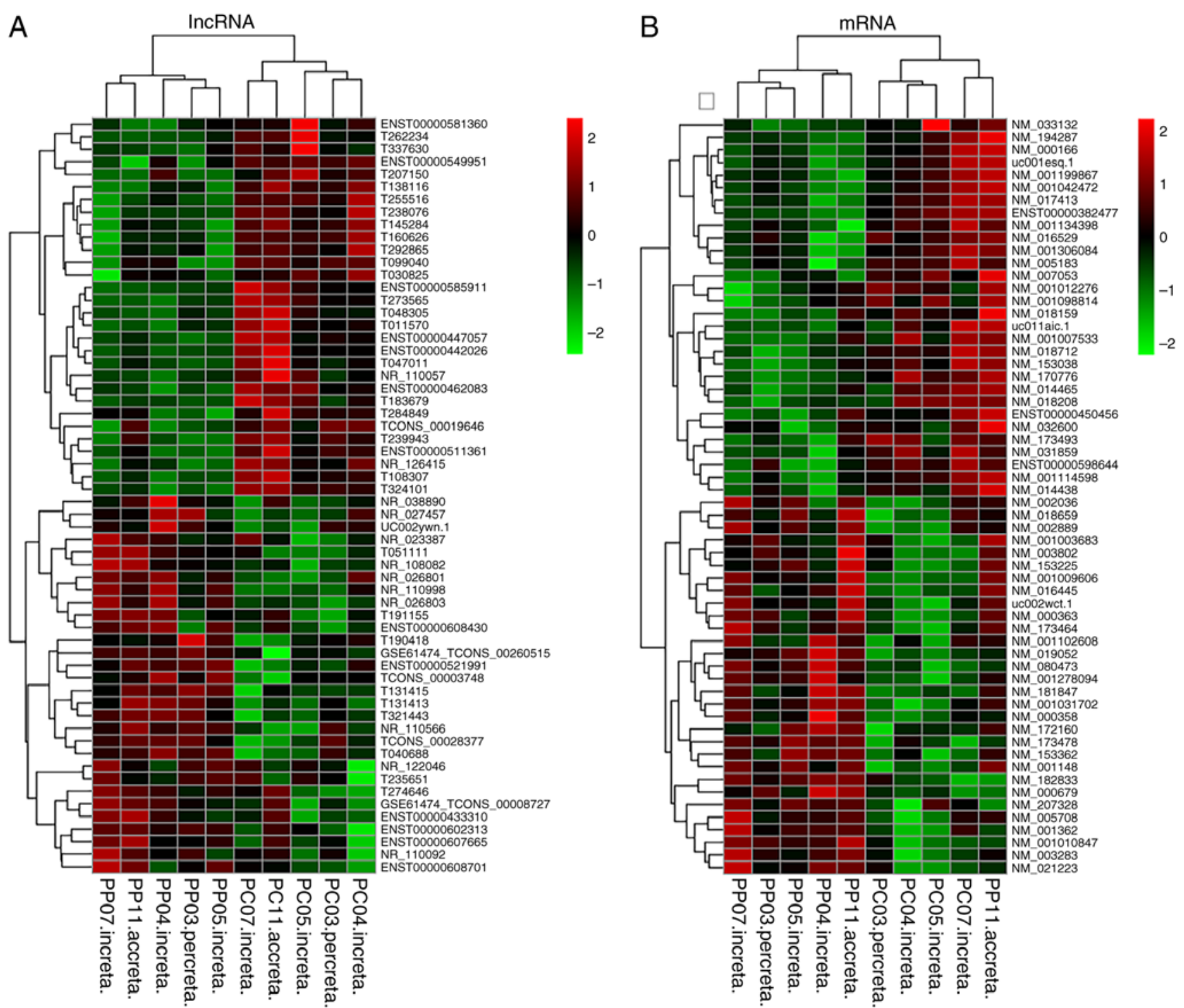

Figure 1. Differentially expressed (A) lncRNAs and (B) mRNAs between the invasive and control groups. Expression values are represented as red and green, which indicate upregulation and downregulation, respectively. IncRNAs or mRNAs with an expression fold change $>1.5$ and with a false discovery rate-adjusted P-value $<0.05$ were considered statistically significant. Invasive group: Pp03, Pp04, Pp05, Pp07 and Pp11; control group: Pc03, Pc04, Pc05, Pc07 and Pc11. lncRNA, long non-coding RNA.

process (GO:0032501) and receptor binding (GO:0005102), respectively.

Pathway analysis was performed by mapping genes to KEGG pathways. The 10 most significantly enriched pathways corresponding to dysregulated mRNAs are listed in Fig. 4B. The most enriched pathways were the TGF- $\beta$ signaling pathway (KEGG ID: hsa04350) for upregulated mRNAs and the pentose phosphate pathway (KEGG ID: hsa00030) for downregulated mRNAs.

\section{Discussion}

The present study provided a comprehensive analysis of lncRNA and mRNA expression profiles in AIP. In invasive placenta tissues, a total of 329 lncRNAs (101 upregulated; 228 downregulated) and 179 mRNAs (95 upregulated; 84 downregulated) were differentially expressed compared with adherent noninvasive tissues. Most of the dysregulated lncRNAs were from intergenic regions (72.15\%). The RT-qPCR results are consistent with the results of the microarray assays, proving the reliability of the microarray results.

GO analysis indicated that the top three functions of all dysregulated genes were enriched in proteinaceous ECM, ECM and ECM components. Physiologically, ECM provides biochemical and structural support to surrounding cells (17) and undergoes vigorous reorganization in the process of placenta implantation (18). ECM components are critical for trophoblast invasion (19). Heparan sulfate proteoglycans, an ample ECM component of the placenta, may decentralize the ECM and promote trophoblast invasion to the myometrium when they degrade (19). Other ECM components, such as decorin and biglycan, were reported to be involved in placental invasion (20). In addition, ECM elasticity may direct cellular differentiation, including the epithelial-mesenchymal transition (EMT), and studies have indicated that trophoblast EMT has an important role in AIP $(21,22)$. MMPs in the ECM are able remodel the ECM to facilitate EMT, promoting cell specification during embryonic and placental development $(23,24)$. 
Table II. Coexpression analysis of dysregulated lncRNAs subgroup.

\begin{tabular}{|c|c|c|c|c|c|}
\hline Gene symbol & $\begin{array}{l}\text { Fold change- } \\
\text { lncRNAs }\end{array}$ & $\begin{array}{l}\text { Genome } \\
\text { relationship }\end{array}$ & $\begin{array}{l}\text { Nearby } \\
\text { gene symbol }\end{array}$ & $\begin{array}{l}\text { Nearby } \\
\text { protein name }\end{array}$ & $\begin{array}{l}\text { Fold change } \\
\text {-mRNAs }\end{array}$ \\
\hline CTD-2587H24.10 & -2.73297 & Upstream & TNNI3 & Troponin I type 3 (cardiac) & +1.88938 \\
\hline CTD-2587H24.10 & -2.73297 & Upstream & TNNT1 & Troponin T type 1 (skeletal, slow) & +1.983701 \\
\hline VTRNA2-1 & -1.53625 & Downstream & TGFBI & $\begin{array}{l}\text { Transforming growth factor beta } \\
\text { induced }\end{array}$ & +1.983147 \\
\hline G008916 & +1.677219 & Upstream & BAMBI & $\begin{array}{l}\text { BMP and activin membrane-bound } \\
\text { inhibitor }\end{array}$ & +1.557582 \\
\hline G023160 & +1.567031 & Downstream & ZIC5 & Zic family member 5 & -2.799566 \\
\hline G037467 & -1.617928 & Downstream & ACAA2 & Acetyl-CoA acyltransferase 2 & -1.680578 \\
\hline G045620 & -1.640229 & Upstream & PCDP1 & Primary ciliary dyskinesia 1 homolog & -2.004573 \\
\hline G052555 & +1.570389 & Upstream & ADARB1 & Adenosine deaminase, RNA-specific, B1 & +1.525046 \\
\hline G052559 & -1.515549 & Downstream & ADARB1 & Adenosine deaminase, RNA-specific, B1 & +1.525046 \\
\hline G052938 & -1.686997 & Downstream & GGT2 & Gamma-glutamyltransferase 2 & -1.921371 \\
\hline RP11-247A12.1 & -1.716386 & Natural antisense & CRAT & Carnitine O-acetyltransferase & -1.592573 \\
\hline AK057317 & -1.758716 & Intronic antisense & ASIC2 & Acid sensing ion channel subunit 2 & +1.618979 \\
\hline
\end{tabular}

lncRNA, long non-coding RNA. VTRNA2-1, vault RNA2-1.
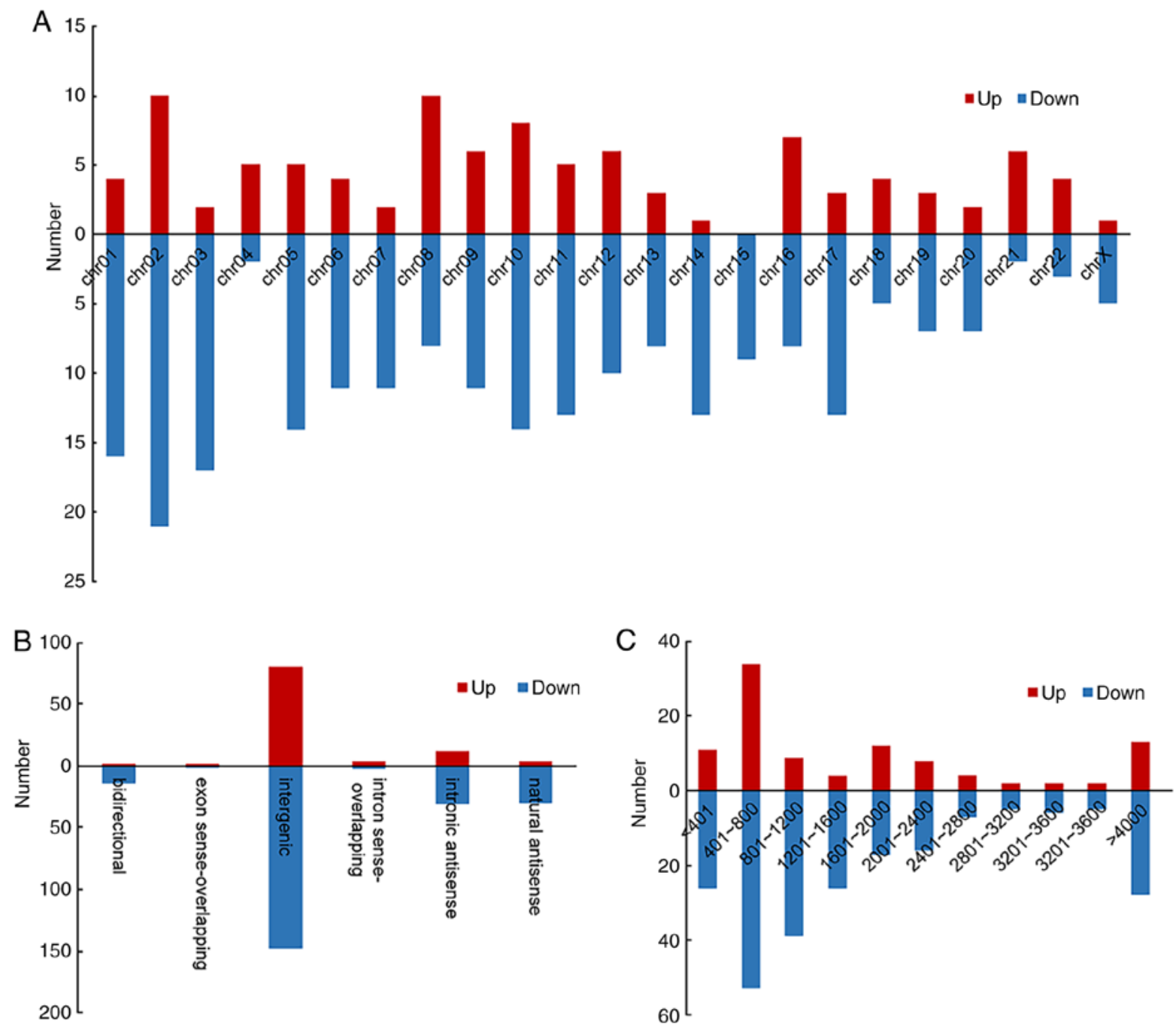

Figure 2. Expression signatures of the dysregulated lncRNAs between the invasive group and the control group. (A) Chromosome distribution of lncRNAs. Chromosomes 2 and 10 had the highest number of dysregulated lncRNAs. (B) Classification of lncRNAs. They were mainly intergenic. (C) Length distribution of lncRNAs (nt). They were mainly between 401 and 800 nt in length. lncRNA, long non-coding RNA; chr, chromosome. 

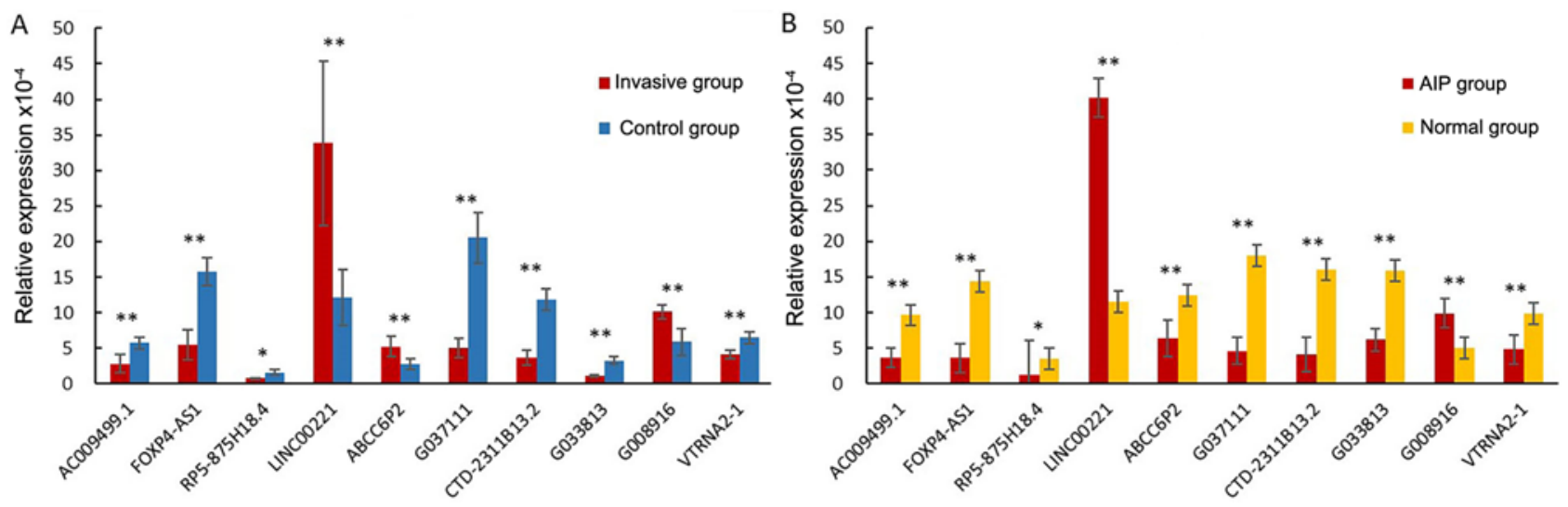

C

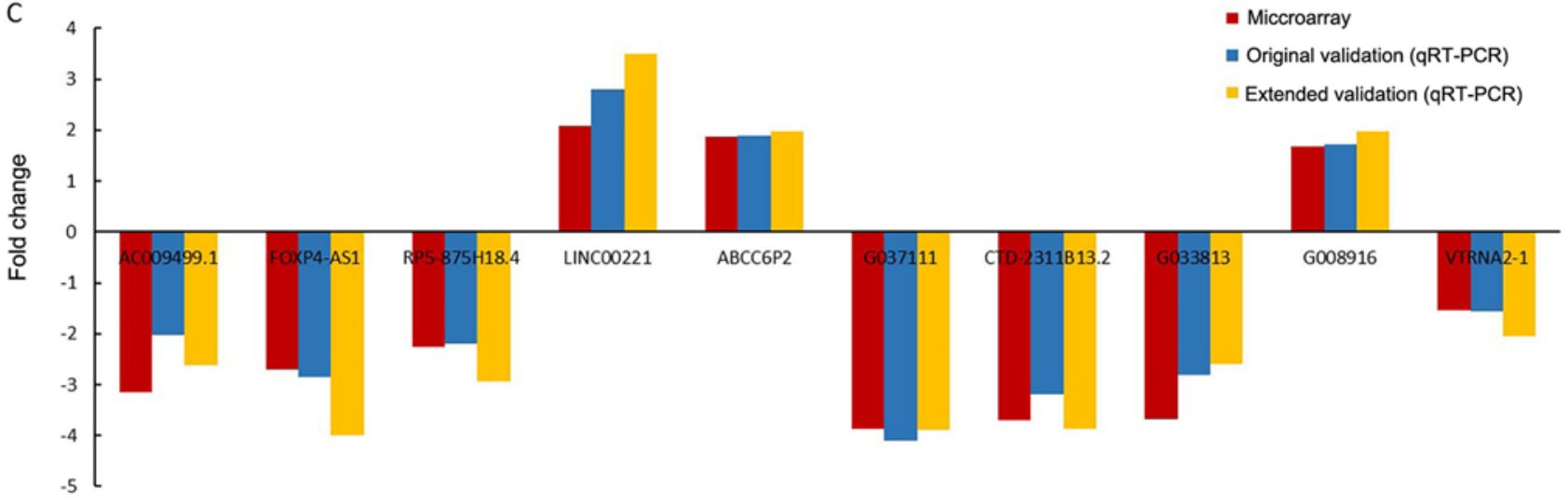

Figure 3. Validation of the microarray data by RT-qPCR. (A and B) Relative expression levels of 10 dysregulated lncRNAs. (A) Original validation. Five paired samples were collected form the 5 patients with AIP in the invasive site and adherent noninvasive site. Samples were divided into invasive group and control group. Values are expressed as the mean \pm standard error. ${ }^{*} \mathrm{P}<0.05,{ }^{* *} \mathrm{P}<0.01$, paired Mann-Whitney U tests. (B) extended validation. Samples were divided into AIP group and normal group. Samples of AIP were collected from 15 patients with AIP. Samples of normal group were collected from 15 patients with a normal placenta. Values are expressed as the mean \pm standard error. ${ }^{*} \mathrm{P}<0.05,{ }^{* *} \mathrm{P}<0.01$, Mann-Whitney $\mathrm{U}$ tests. (C) Comparison of the microarray data, RT-qPCR validation and extended RT-qPCR validation. The 10 PCR-validated lncRNAs in the original and extended samples exhibited similar fold-change tendencies to those observed in the microarray results. IncRNA, long non-coding RNA; RT-qPCR, reverse transcription-quantitative PCR.

Furthermore, placental basement membrane proteins also participate in trophoblast invasion; they constitute $>80 \%$ of ECM proteins in the placental basal plate and are indispensable for effective cytotrophoblast invasion (25). As the results of the present functional enrichment analysis indicate, the basement membrane had the fifth-highest enrichment score, which is consistent with the above results. The ECM is derived from a multicellular organismal process, which has important functions in cell adhesion, differentiation and cell-to-cell communication (26). These different roles may be the reason why the category of multicellular organismal process has the fourth-highest enrichment score. KEGG pathway analysis for dysregulated mRNAs revealed that the top enriched pathway was the TGF- $\beta$ signaling pathway. According to previous studies, numerous cellular processes, including EMT (27-29), ECM neogenesis and placenta formation are regulated by the TGF- $\beta$ signaling pathway (30). The results of the KEGG analysis of the present study were consistent with those of the GO enrichment analysis. Furthermore, Duzyj et al (31) indicated that the endoglin-TGF- $\beta$ pathway system is abnormally expressed in the invasive site of the placenta, which increases the invasiveness of placental cells. While no previous IncRNA studies have been performed on the regulation of AIP, to the best of our knowledge, the present study provided clues that dysregulated lncRNAs may regulate the process of ECM neogenesis and placenta formation to facilitate the process of AIP through the TGF- $\beta$ signaling pathway.

Previous research has demonstrated that lncRNAs are able to regulate the expression of nearby mRNAs (32). A coexpression analysis performed in the present study suggested that the expression of 10 lncRNAs and 2 antisense lncRNAs was correlated with the expression of nearby mRNAs. Although the corresponding functions of these mRNAs in AIP remain elusive, based on previous studies and GO analysis, it was indicated that they are mainly involved in muscle contraction regulation [troponin I1 (TNNI1), troponin I3 (TNNI3)] (33), TGF- $\beta$ signaling [BMP and activin membrane-bound inhibitor (BAMBI) and TGF- $\beta$-induced (TGFBI)] $(34,35)$, cell proliferation, migration, aggressiveness [ZIC5, acetyl-CoA acyltransferase 2 (ACAA2)] (36-39), multicellular organism function [adenosine deaminase RNA specific B1 (ADARB1), acid sensing ion channel subunit 2 (ASIC2)], amino acid regulation [gamma-glutamyltransferase 2 (GGT2)] and fat metabolism [carnitine O-acetyltransferase (CRAT)]. Of note, BAMBI and TGFBI, as the target genes of the TGF- $\beta$ pathway (34), are closely related to the ECM and invasive placental cells, and their nearby lncRNAs G008916 and VTRNA2-1 were upregulated and downregulated, respectively. 
A

Proteinaceous extracellular matrix -

Extracellular matrix -

Extracellular matrix component -

Multicellular organismal process -

Basement membrane -

Extracellular region part -

Second-messenger-mediated signaling -

Multicellular organism development -

Receptor binding -

Muscle contraction -
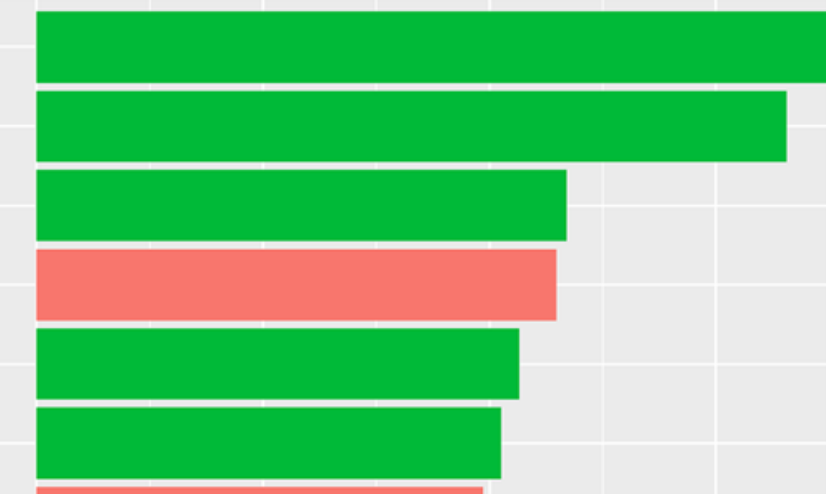

$$
\text { 西 }
$$
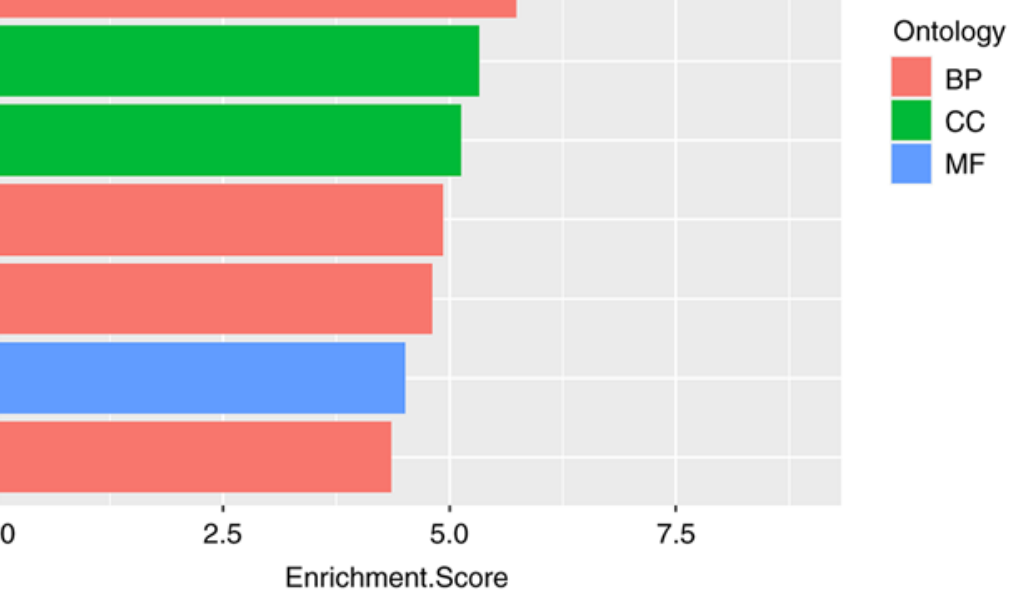

B TGF- $\beta$ signaling pathway -
Pentose phosphate pathway -

Fructose and mannose metabolism -

Protein digestion and absorption -

Calcium signalling pathway -

Glycolysis/gluconeogenesis -

CAMP signaling pathway -

Aldosterone synthesis and secretion -

Insulin secretion -

Adrenergic signaling in cardiomyocytes -

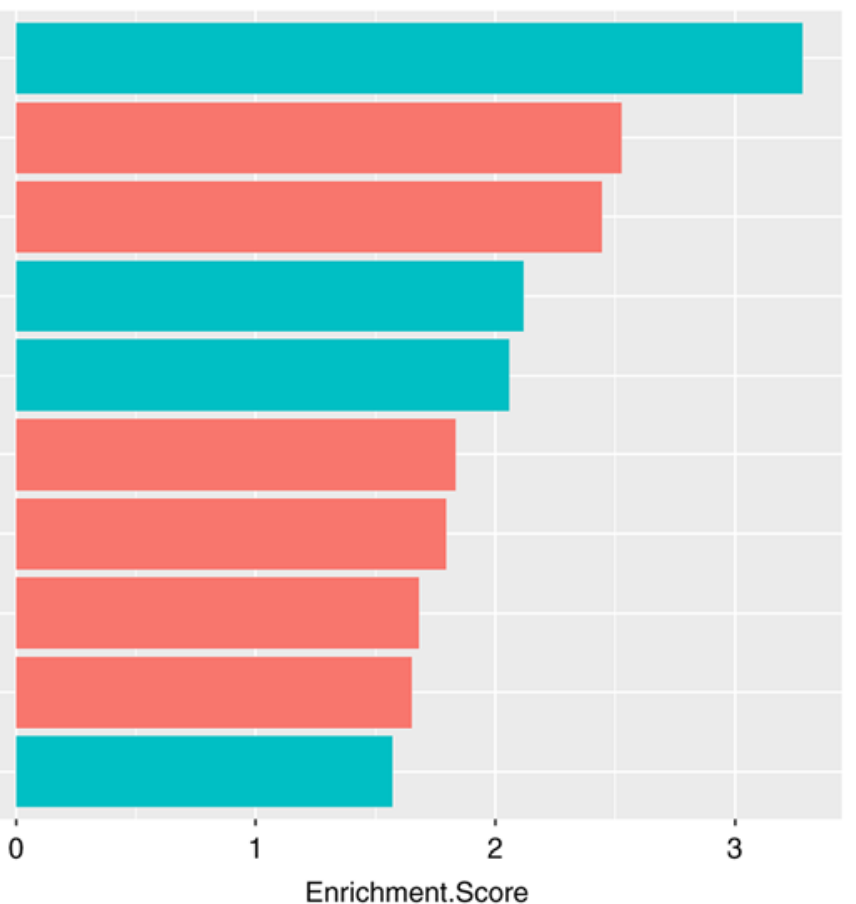

Regulation

Rown

Up

Enrichment.Score

Figure 4. GO and KEGG analysis. (A) GO enrichment analysis of mRNAs. All of the 10 most significantly enriched functions were enriched by upregulated mRNAs. The most enriched function was related to the proteinaceous extracellular matrix. (B) KEGG pathway analysis. The most significantly enriched pathway was the TGF- $\beta$ signaling pathway. GO, gene ontology; KEGG, Kyoto Encyclopaedia of Genes and Genomes; BP, biological process; CC, cellular component; MF, molecular function.

According to the results of the subgroup and the GO and KEGG analyses, it is indicated that BAMBI and TGFBI have important roles in AIP through TGF- $\beta$ pathway to promote placental invasion.

The present study has certain strengths and limitations. The present study was the first to explore the lncRNAs expression profile in patients with AIP. The present study identified and validated certain dysregulated lncRNAs in AIP. However, there are certain limitations to this study. One major limitation was that no normal group was used for comparison with the invasive group and control group when performing lncRNA microarray scanning, as both of them were sampled from patients with AIP. Using a normal group with samples from patients without AIP may make the comparison more complete. In order to remedy this, extended sample validation was performed and the results of the extended qPCR validation were consistent with the original sample validation and microarray data. 
In summary, the dysregulated mRNAs were mainly enriched in functional terms associated with the extracellular matrix and in the TGF- $\beta$ signaling pathway. IncRNAs G008916 and VTRNA2-1 corresponded to the nearby mRNAs BAMBI and TGFBI, which were both involved in the TGF- $\beta$ pathway and were strictly associated with the ECM and the invasion of placental cells. These lncRNAs have great potential to serve as biomarkers of AIP.

\section{Acknowledgements}

The authors thank the obstetricians Dr Yan Wang and Dr Meng Zeng (all, Department of Obstetrics, Foshan Women and Children's Hospital Affiliated to Southern Medical University) for helping with the collection of the placenta tissues.

\section{Funding}

No funding was received.

\section{Availability of data and materials}

The expression data were stored in the Gene Expression Omnibus database (https://www.ncbi.nlm.nih.gov/geo; accession no. GSE126552).

\section{Authors' contributions}

ZL, SY, SW, HM and HZ designed the experiments. ZL decided the final experimental scheme. SY collected and analysed the data. SW and HM interpreted the data. HZ analysed the data and drafted the manuscript. All authors read and approved the final manuscript.

\section{Ethics approval and consent to participate}

The Human Ethics Committee of the Foshan Women and Children's Hospital Affiliated to Southern Medical University approved the present study (approval no.FSFY-MEC-2017-055). Written informed consent was obtained from all pregnant females for using their specimens and clinical information.

\section{Patient consent for publication}

Not applicable.

\section{Competing interests}

The authors declare that they have no competing interests.

\section{References}

1. Baldwin HJ, Patterson JA, Nippita TA, Torvaldsen S, Ibiebele I, Simpson JM and Ford JB: Antecedents of abnormally invasive placenta in primiparous women: Risk Associated with gynecologic procedures. Obstet Gynecol 131: 227-233, 2018.

2. Baldwin HJ, Patterson JA, Nippita TA, Torvaldsen S, Ibiebele I, Simpson JM and Ford JB: Maternal and neonatal outcomes following abnormally invasive placenta: A population-based record linkage study. Acta Obstet Gynecol Scand 96: 1373-1381, 2017.
3. Iacovelli A, Liberati M, Khalil A, Timor-Trisch I, Leombroni M, Buca D, Milani M, Flacco ME, Manzoli L, Fanfani F, et al: Risk factors for abnormally invasive placenta: A systematic review and meta-analysis. J Matern Fetal Neonatal Med 33: 471-481, 2020.

4. Nieto-Calvache AJ, López-Girón MC, Quintero-Santacruz M, Bryon AM, Burgos-Luna JM, Echavarría-David MP, López L, Macia-Mejia C and Benavides-Calvache JP: A systematic multidisciplinary initiative may reduce the need for blood products in patients with abnormally invasive placenta. J Matern Fetal Neonatal Med: 1-7, 2020.

5. Erfani H, Fox KA, Clark SL, Rac M, Rocky Hui SK, Rezaei A, Aalipour S, Shamshirsaz AA, Nassr AA, Salmanian B, et al: Maternal outcomes in unexpected placenta accreta spectrum disorders: Single-center experience with a multidisciplinary team. Am J Obstet Gynecol 221: 337.e1-337.e5, 2019.

6. Collins SL, Alemdar B, van Beekhuizen HJ, Bertholdt C, Braun T, Calda P, Delorme P, Duvekot JJ, Gronbeck L, Kayem G, et al: Evidence-based guidelines for the management of abnormally invasive placenta: Recommendations from the international society for abnormally invasive placenta. Am J Obstet Gynecol 220: 511-526, 2019.

7. Buca D, Liberati M, Cali G, Forlani F, Caisutti C, Flacco ME, Manzoli L, Familiari A, Scambia G and D'Antonio F: Influence of prenatal diagnosis of abnormally invasive placenta on maternal outcome: Systematic review and meta-analysis. Ultrasound Obstet Gynecol 52: 304-309, 2018.

8. Shainker SA, Silver RM, Modest AM, Hacker MR, Hecht JL, Salahuddin S, Dillon ST, Ciampa EJ, Dalton ME, Otu HH, et al: Placenta accreta spectrum: Biomarker discovery using plasma proteomics. Am J Obstet Gynecol 223: 433.e1-433.e14, 2020.

9. Al-Khan A, Youssef YH, Feldman KM, Illsley NP, Remache Y, Alvarez-Perez J, Mannion C, Alvarez $M$ and Zamudio S: Biomarkers of abnormally invasive placenta. Placenta 91: 37-42, 2020.

10. Jauniaux E, Bhide A, Kennedy A, Woodward P, Hubinont C and Collins S; FIGO Placenta Accreta Diagnosis and Management Expert Consensus Panel: FIGO consensus guidelines on placenta accreta spectrum disorders: Prenatal diagnosis and screening. Int J Gynaecol Obstet 140: 274-280, 2018.

11. Kopp F and Mendell J: Functional classification and experimental dissection of long noncoding RNAs. Cell 172: 393-407, 2018

12. McAninch D, Roberts CT and Bianco-Miotto T: Mechanistic insight into long noncoding RNAs and the placenta. Int J Mol Sci 18: 1371, 2017.

13. Tseng JJ, Hsieh YT, Hsu SL and Chou MM: Metastasis associated lung adenocarcinoma transcript 1 is up-regulated in placenta previa increta/percreta and strongly associated with trophoblast-like cell invasion in vitro. Mol Hum Reprod 15: 725-731, 2009.

14. Jauniaux E, Chantraine F, Silver RM and Langhoff-Roos J; FIGO Placenta Accreta Diagnosis and Management Expert Consensus Panel: FIGO consensus guidelines on placenta accreta spectrum disorders: Epidemiology. Int J Gynaecol Obstet 140: 265-273, 2018.

15. Jauniaux E and Ayres-de-Campos D; FIGO Placenta Accreta Diagnosis and Management Expert Consensus Panel: FIGO consensus guidelines on placenta accreta spectrum disorders: Introduction. Int J Gynaecol Obstet 140: 261-264, 2018.

16. Mazzei M, Vascellari M, Zanardello C, Melchiotti E, Vannini S, Forzan M, Marchetti V, Albanese F and Abramo F: Quantitative real time polymerase chain reaction (qRT-PCR) and RNAscope in situ hybridization (RNA-ISH) as effective tools to diagnose feline herpesvirus-1-associated dermatitis. Vet Dermatol 30: 491-e147, 2019.

17. Michel G, Tonon T, Scornet D, Cock JM and Kloareg B: The cell wall polysaccharide metabolism of the brown alga Ectocarpus siliculosus. Insights into the evolution of extracellular matrix polysaccharides in Eukaryotes. New Phytol 188: 82-97, 2010.

18. Graubner FR, Boos A, Aslan S, Kücükaslan I and Kowalewski MP: Uterine and placental distribution of selected extracellular matrix (ECM) components in the dog. Reproduction 155: 403-421, 2018

19. Haimov-Kochman R, Friedmann Y, Prus D, Goldman-Wohl DS, Greenfield C, Anteby EY, Aviv A, Vlodavsky I and Yagel S: Localization of heparanase in normal and pathological human placenta. Mol Hum Reprod 8: 566-573, 2002.

20. Borbely AU, Daher S, Ishigai MM, Mattar R, Sun SY, Knöfler M, Bevilacqua E and Oliveira SF: Decorin and biglycan immunolocalization in non-villous structures of healthy and pathological human placentas. Histopathology 64: 616-625, 2014. 
21. DaSilva-Arnold SC, Zamudio S, Al-Khan A, Alvarez-Perez J Mannion C, Koenig C, Luke D, Perez AM, Petroff M, Alvarez M and Illsley NP: Human trophoblast epithelial-mesenchymal transition in abnormally invasive placenta. Biol Reprod 99: 409-421, 2018.

22. Thiery JP and Sleeman JP: Complex networks orchestrate epithelial-mesenchymal transitions. Nat Rev Mol Cell Biol 7: 131-142, 2006.

23. Hiden U, Eyth CP, Majali-Martinez A, Desoye G, Tam-Amersdorfer C, Huppertz B and Ghaffari Tabrizi-Wizsy N: Expression of matrix metalloproteinase 12 is highly specific for non-proliferating invasive trophoblasts in the first trimester and temporally regulated by oxygen-dependent mechanisms including HIF-1A. Histochem Cell Biol 149: 31-42, 2018.

24. Nguyen AH, Wang Y, White DE, Platt MO and McDevitt TC: MMP-mediated mesenchymal morphogenesis of pluripotent stem cell aggregates stimulated by gelatin methacrylate microparticle incorporation. Biomaterials 76: 66-75, 2016.

25. Kuo CY, Guo T, Cabrera-Luque J, Arumugasaamy N, Bracaglia L, Garcia-Vivas A, Santoro M, Baker H, Fisher J and Kim P: Placental basement membrane proteins are required for effective cytotrophoblast invasion in a three-dimensional bioprinted placenta model. J Biomed Mater Res A 106: 1476-1487, 2018

26. Abedin M and King N: Diverse evolutionary paths to cell adhesion. Trends Cell Biol 20: 734-742, 2010.

27. Miyazono K, Katsuno Y, Koinuma D, Ehata S and Morikawa M: Intracellular and extracellular TGF- $\beta$ signaling in cancer: Some recent topics. Front Med 12: 387-411, 2018.

28. Huang TW, Li ST, Fang KM and Young TH: Hyaluronan antagonizes the differentiation effect of TGF- $\beta 1$ on nasal epithelial cells through down-regulation of TGF- $\beta$ type I receptor. Artif Cells Nanomed Biotechnol 46 (Suppl 3): S254-S263, 2018.

29. Rocha MR, Barcellos-de-Souza P, Sousa-Squiavinato ACM, Fernandes PV, de Oliveira IM, Boroni M and Morgado-Diaz JA: Annexin A2 overexpression associates with colorectal cancer invasiveness and TGF- $\beta$ induced epithelial mesenchymal transition via Src/ANXA2/STAT3. Sci Rep 8: 11285, 2018.

30. Prossler J, Chen Q, Chamley L and James JL: The relationship between TGF $\beta$, low oxygen and the outgrowth of extravillous trophoblasts from anchoring villi during the first trimester of pregnancy. Cytokine 68: 9-15, 2014.
31. Duzyj CM, Buhimschi IA, Laky CA, Cozzini G, Zhao G, Wehrum M and Buhimschi CS: Extravillous trophoblast invasion in placenta accreta is associated with differential local expression of angiogenic and growth factors: A cross-sectional study. BJOG 125: 1441-1448, 2018.

32. Ørom UA, Derrien T, Beringer M, Gumireddy K, Gardini A, Bussotti G, Lai F, Zytnicki M, Notredame C, Huang Q, et al: Long noncoding RNAs with enhancer-like function in human cells. Cell 143: 46-58, 2010.

33. Sheng JJ and Jin JP: TNNI1, TNNI2 and TNNI3: Evolution, regulation, and protein structure-function relationships. Gene 576: 385-394, 2016.

34. Ge J, Apicella M, Mills JA, Garcon L, French DL, Weiss MJ, Bessler M and Mason PJ: Dysregulation of the transforming growth factor $\beta$ pathway in induced pluripotent stem cells generated from patients with diamond blackfan anemia. PLoS One 10: e0134878, 2015.

35. Guo SK, Shen MF, Yao HW and Liu YS: Enhanced expression of TGFBI promotes the proliferation and migration of glioma cells. Cell Physiol Biochem 49: 1097-1109, 2018.

36. Li GF, Li L, Yao ZQ and Zhuang SJ: Hsa_circ_0007534/ miR-761/ZIC5 regulatory loop modulates the proliferation and migration of glioma cells. Biochem Biophys Res Commun 499: 765-771, 2018 .

37. Nyholm MK, Wu SF, Dorsky RI and Grinblat Y: The zebrafish zic2a-zic5 gene pair acts downstream of canonical Wnt signaling to control cell proliferation in the developing tectum. Development 134: 735-746, 2007.

38. Satow R, Nakamura T, Kato C, Endo M, Tamura M, Batori R, Tomura S, Murayama Y and Fukami K: ZIC5 drives melanoma aggressiveness by PDGFD-mediated activation of FAK and STAT3. Cancer Res 77: 366-377, 2017.

39. Yang Y, Fang X, Yang R, Yu H, Jiang P, Sun B and Zhao Z: MiR-152 regulates apoptosis and triglyceride production in MECs via targeting ACAA2 and HSD17B12 genes. Sci Rep 8: 417, 2018.

This work is licensed under a Creative Commons

Attribution-NonCommercial-NoDerivatives 4.0 International (CC BY-NC-ND 4.0) License. 